\title{
Problems of defining length
}

SIR - Asher Peres ${ }^{1}$ discusses three aspects of length measurement: the definition of the SI unit of length, the realization of that unit, and the use of the unit to perform a measurement, and touches on problems that we have already discussed 2,3 .

The relevant postulates of Einstein's relativity theory have been established experimentally with an accuracy well beyond that of the present best methods of realizing the SI metre; particularly so for Michelson-Morley experiments ${ }^{4}$. The nature of length has not been detectably changed by the new definition, nor has the size of the SI unit. The Conference Générale des Poids et Mesures (CGPM) has not recommended any changes in the laws required for scaling up or down from the unit length in order to make length measurements, neither is one obliged to use light for this purpose. The unit is merely a reproducible scalar representation of the physical quantity against which other lengths may be compared. The only change is the manner in which the new definition is specified and realized. The velocity of light is not asserted to be constant, but if it is changing, then the size of at least one of the SI units will be changing also - relative to some absolute units to which we do not yet have access (the units of gedanken metrology). Thus for cosmological experiments, where the constancy of the unit is required over distances and times, comparable with the size and age of the universe, respectively, neither the SI units nor the natural units (e.g. where $e=m_{e}$ $=c=1$ ) can necessarily be relied on. The SI units are of a pragmatic nature, and are designed to provide the most invariant units that can be achieved with the science and technology of the day: if the present methods become detectably inadequate they will be replaced by something better.

The definition of a unit tells us little about the nature of the physical quantity concerned, and we need other instructions in order to know how to measure a physical quantity. The definition of the kilogram, for example, is clearly not intended to define the concept of mass, or to instruct on how masses should be intercompared. Similarly the definition of the metre is not intended to be a definition of what we mean by length, or of how lengths must be measured. The CGPM has redefined the SI unit of length, but not the meaning of length itself.

The CGPM provides a definition of the unit ${ }^{5}$ which gives sufficient information for the great majority of users. The definition is accompanied by a mise en pratique which contains information and recommendations concerning the manner by which the unit should be realized if the full accuracy is required. The onus is on any metrologist who chooses to use a different method to demonstrate that the unit so realized is of the same magnitude as that produced by the recommended methods moreover, it is stated that proper use must be made of the state of knowledge of the day in applying corrections for known effects (whether electromagnetic, quantum-electrodynamic or relativistic effects, and so on). Professor Peres is correct in stating that although the new definition might be thought to require two synchronized clocks, the practicality is that the round trip time is measured and divided by two. This is indeed the current practice in both interferometry and time of flight measurements (such as lunar ranging) and is implicit in the mise en pratique.

The CGPM recommendations are the result of extensive consultations with scientists and technologists throughout the world, but they cannot anticipate future discoveries other than by extrapolating existing trends. For example, if there were found to be small, but significant, anisotropy in the velocity of light (that is some preferred axis in space) then this property would either be incorporated in the definition itself or in the mise en pratique. At present we know that the new definition can be realized with a considerably greater accuracy than could be achieved with the former krypton- 86

\section{English at risk}

SIR - Your invitation (Nature 306, 134; 1983) to readers for comments on the abuse of language seems to have evoked little response. May I reopen the issue?

Idso and Brazel (Nature 312, 51; 1984) write: "Inclusion of this latter effect in a simple model of watershed runoff applied to 12 drainage basins in Arizona..." Various points made in the letter, and the North American addresses of the authors, suggest that by watershed they mean a catchment area.

So what distinction is being made in the quoted passage? Or is it just what Fowler and Fowler called "elegant variation"? I have already pointed out (Nature 307, 312; 1984) that the meaning of watershed is so uncertain that it is unsuitable for use in the mixed company of an international readership.

A new verbal abuse is that the distinction between credibility and credence is in danger. A statement has credibility, credence is something given to it by a human mind. Nature is concerned with the written rather than the spoken word. May I however grumble about the error, often heard in radio science programmes, of pronouncing dissect as if were spelt diesect. The word means "to cut apart" not "to cut in two", and one of the few almost unbroken rules of English spelling is that a double consonant shortens the preceding vowel.

N.W. PIRIE 42 Leyton Road, Harpenden, Herts, UK realization of the metre.

On the question of the use of the unit, Professor Peres uses light to measure the circumference of the Earth and so it follows that it must be in some form of waveguide in order to circumnavigate the Earth. In such a case it would, of course, be necessary to take account of a number of effects and to make the appropriate corrections, including that for the rotation of the Earth.

Relativistic effects can be encountered with measurements of mass, length, time, in electromagnetism and elsewhere, however their base units are defined. As accuracies improve, it will be necessary for more and more people to take these and other sophisticated effects into account before interpreting the results of measurements. It is certainly important to ensure that the units of experimental and theoretical sciences remain conceptually consistent whenever changes are made.

B.W. PETLEY

W.R.C. ROWLEY

National Physical Laboratory, Teddington, Middlesex TWII OLW, UK

1. Peres, A. Nature 312, 10 (1984)

2. Petley, B.W. Nature 303, 373-376 (1983).

3. Rowley, W.R.C. Phys. Bull. 36, 282-284 (1984).

4. Brillet, A. \& Hall, J.A. Phys. Rev. Lett. 42, 549553 (1979).

5. Metrologia 19, 163-178 (1984)

\section{Exosat}

SIR - Your news item "Britain in space strong links with Europe urged" (8 November, p.92) refers to "some recent failures of instruments built for other people's satellites (such as some of the detectors for the Exosat satellite)". We should like to point out that the Exosat detectors which have failed were not built by a British space research group but by industrial contractors selected by the European Space Agency. The early development of the prototype detectors was carried out in this laboratory, but, contrary to previous practice, none of the flight hardware was built here. This point seems to have escaped many people's attention.

J.L. Culhane

Mullard Space Science Laboratory,

University College London,

Holmbury St Mary, Dorking,

Surrey RH5 6NT, UK

\section{Not our drugs}

SIR - I write to draw attention to a substantial mistake in your leading article "What price drugs?"' (15 November p. 184)).

The drugs Valium and Mogadon are produced by Roche Products Limited and not by Ciba-Geigy Pharmaceuticals; we are in no way involved with their production or marketing

Ciba-Geigy Rbarmaceuticals,

Wimblehurst isoad,

Horsham, West Sussex RH12 4AB 